\title{
PASSIVE MICROFLOW REGULATION USING THERMALLY RESPONSIVE POLYMERS
}

\author{
Boris Stoeber \\ Departments of Mechanical and Electrical \& Computer Engineering, University of British Columbia \\ Vancouver, BC, CANADA
}

\author{
Dorian Liepmann \\ Department of Bioengineering \\ University of California \\ Berkeley, CA, U.S.A.
}

\author{
Susan J. Muller \\ Department of Chemical Engineering \\ University of California \\ Berkeley, CA, U.S.A.
}

\begin{abstract}
Thermally responsive polymer solutions have previously been used in microchannels with integrated heaters for active valving. In addition, passive flow regulation based on viscous heating in microchannels can be achieved under constant flow rate and constant pressure drop conditions. In the case of pressuredriven flow through a microchannel, we demonstrate for the first time that viscous heating can cause periodic gel formation leading to pulsatile flow with no need for moving parts or active components. This is a unique concept for passive microflow regulation showing such a performance.
\end{abstract}

\section{INTRODUCTION}

Microflow control is an important field of research driven by the demands of microfluidic applications such as ink-jet printing and lab-on-a-chip devices for biochemical analysis and DNA sequencing. While ink-jet print heads require very fast acting valve mechanisms that operate at the order of $1 \mathrm{kHz}$, the flow control requirements of Micro Total Analysis Systems can be very diverse. In addition to fast and reliable valve actuation, passive flow control or flow control in response to changing environmental variables can be very attractive for such systems in order to efficiently manage biological and chemical samples.

For this purpose, Fréchet, [1], Beebe [2], and others have synthesized temperature, $\mathrm{pH}$, or glucose sensitive polymer structures that are anchored inside microchannels; these materials swell and block the channel in response to changes in the environment. We demonstrate a fundamentally different concept for automatic flow rate control based on the reversible phase change of a thermally responsive polymer present in the flow. Valving occurs in a straight section of a flow channel, where viscous heating triggers the heat-induced gelation of the fluid, which can lead to total blockage of the flow passage or to periodic velocity fluctuations.

\section{RESULTS AND DISCUSSION}

The viscosity $\eta$ of dilute $(\sim 10 \%)$ aqueous solutions of the biocompatible polymer Pluronic F127 (BASF) increases with temperature until the solutions reversibly solidify. This phase change occurs over a temperature change of only $0.5^{\circ} \mathrm{C}$. The gel formation temperature is a function of polymer type and concentration. We previously demonstrated an active microvalve for Pluronic solutions using integrated heaters in microchannels [3]. Here, we demonstrate and explain passive flow control using dilute Pluronic solutions. These mechanisms allow self-regulating valves or valves that respond to changes in ambient temperature.
The simple flow channels (cross section: $100 \mu \mathrm{m} \times 100 \mu \mathrm{m}$ ) used for passive valving were etched into silicon using DRIE and were covered with glass. The high shear rate $\dot{\gamma}=\mathrm{d} v / \mathrm{d} x$ regions along the channel walls can produce significant viscous heating $\Phi=\eta \dot{\gamma}^{2}$. In cases where the ambient temperature is close to the gelation temperature of the Pluronic solution, viscous heating can cause local gel formation on the channel wall. The subsequent flow regime, either channel blockage or velocity fluctuations, depends on the mechanism that drives the flow, controlled flow rate or controlled pressure drop respectively.

In the case of controlled flow rate, gel formation on the channel wall reduces the cross sectional area of the flow channel, which increases the shear rate in the flow. This leads to more viscous heating and to more gel formation until the entire channel is blocked as mentioned in [3].

However, a different mechanism occurs if a controlled pressure drives the flow. Here, the presence of gel on the channel wall increases the flow resistance of the channel, which reduces the flow rate and therefore the shear rate in the flow as shown in Fig. 1. This leads to less viscous heating so that the heat can dissipate into the substrate and the gel becomes liquid again, which can result in flow fluctuations. Figure 2 shows video frames of an $18 \%$ Pluronic solution driven through a microchannel at controlled pressure. The flow gels periodically on the walls and becomes liquid with a time period of $2.2 \mathrm{~s}$.

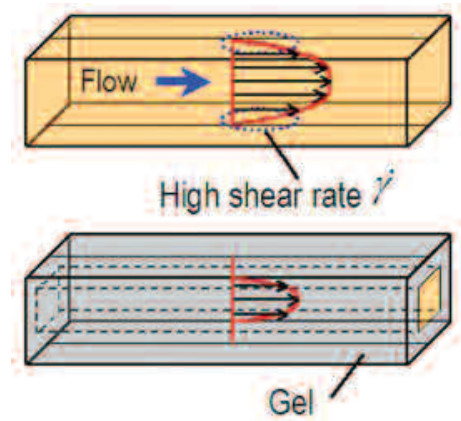

Figure 1. Schematic of gel formation in the channel due to shearinduced viscous heating.

Digital particle image velocimetry (DPIV) results for pressure driven flow (Figs. 3 and 4) show that the flow fluctuations are more pronounced slightly below the gelation temperature $\left(22^{\circ} \mathrm{C}\right)$, while the shear thinning properties of the fluid can lead to a different kind of flow instability above the gel point. The time period of velocity fluctuations from all 18 experiments was $\tau_{p}=2.0 \pm 0.4 \mathrm{~s}$. 


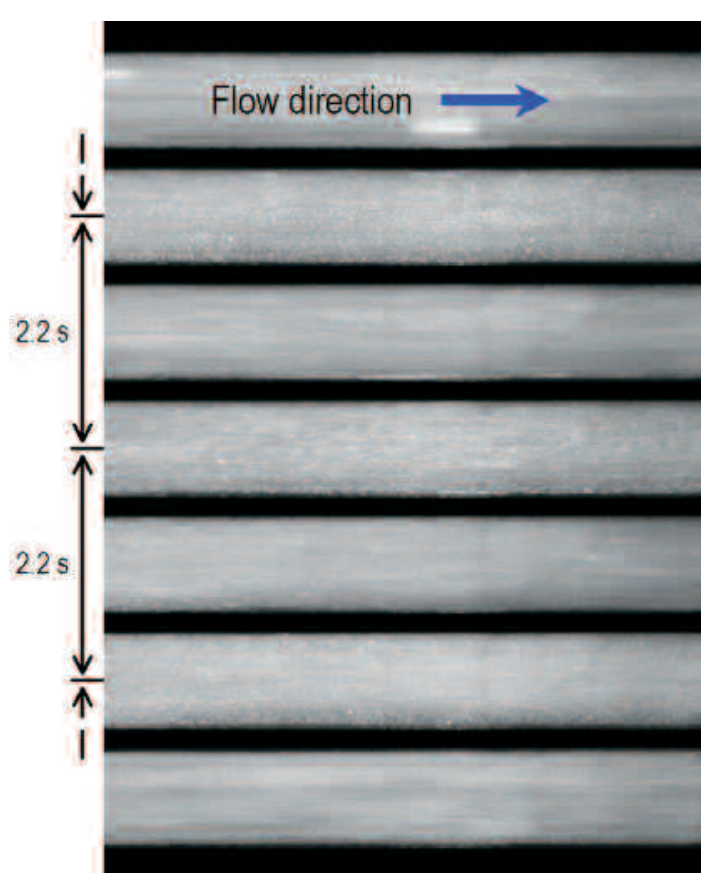

Figure 2. Video frames of seed particles in a Pluronic solution, driven through a microchannel at constant pressure.

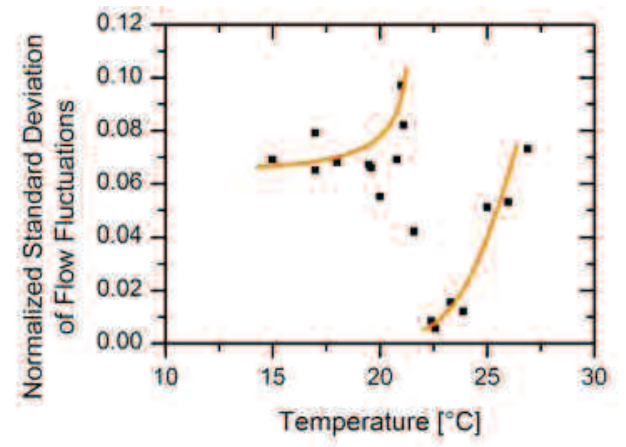

Figure 3. Velocity fluctuations for pressure-driven flow of a Pluronic solution in a microchannel at different temperatures.

This new flow control mechanism is possible because of the particular rheology of Pluronic solutions that are shear-thinning and thermo thickening. In particular, it can be shown that at controlled pressure, the amount of viscous heating as a function of the diameter of the open channel $D$ and the fluid viscosity $\eta$ scales as $\Phi \sim D^{2} / \eta$, while at controlled flow rate viscous heating goes as $\Phi \sim \eta / D^{6}$. A related but fundamentally different kind of shear-induced flow instabilities has been observed previously for the more conventional case of thermo thinning fluids [4].

The velocity profiles in Fig. 4 show the effects of the particular properties of the Pluronic solutions. Below or at the gel formation temperature $\left(15.0^{\circ} \mathrm{C}\right.$ and $\left.21.0^{\circ} \mathrm{C}\right)$ the velocity profile is parabolic suggesting that the solution is still behaving primarily as a Newtonian fluid. Above the gel temperature $\left(22.0^{\circ} \mathrm{C}\right)$ the velocity profile is flattened and appears plug-like showing the effects of shear-thinning along the channel walls. However, at still higher temperatures some seed particles assemble into extended strings of particles on the walls oriented along the channel axis.
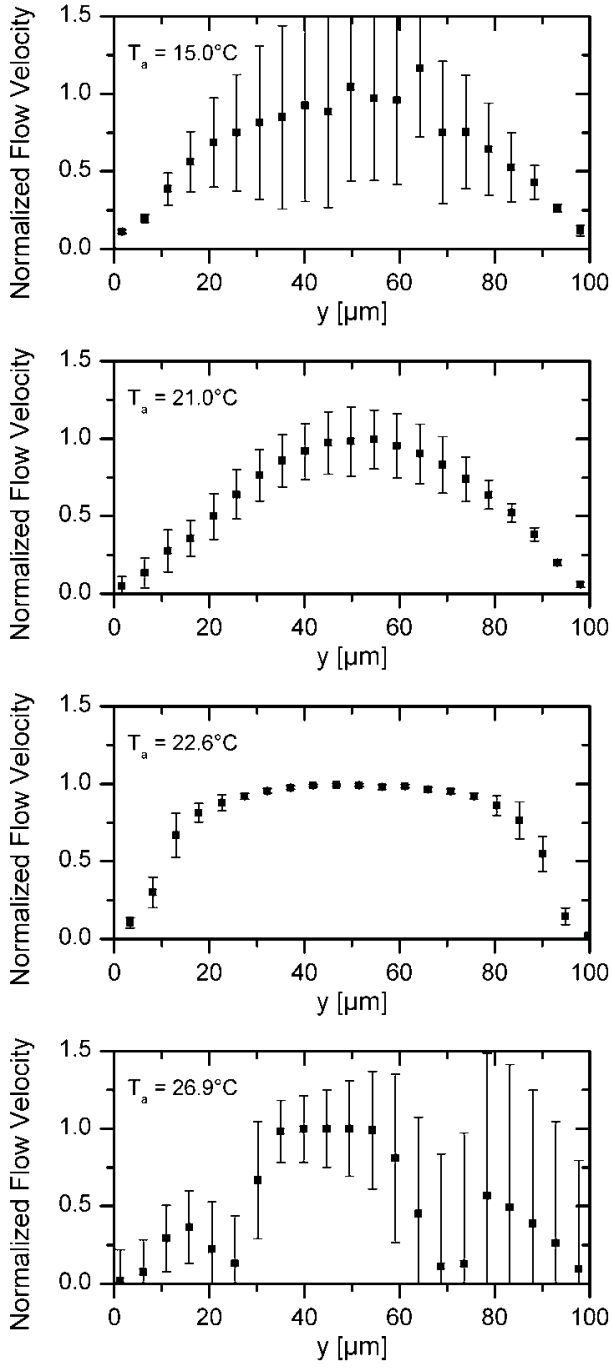

Figure 4. Velocity profiles from DPIV of a Pluronic solution through a microchannel at different ambient temperatures.

\section{CONCLUSIONS}

We demonstrated a new concept for passive microflow regulation that leads to a unique pulsatile flow rate. This can be very useful for Micro Total Analysis Systems in order to effectively manage biological and chemical samples. This concept is mechanically robust because it does not include movable parts. At a fixed temperature, the automatically generated flow fluctuations can be used for controlled dosage of chemical compounds or biological samples transported with the Pluronic solutions. In addition, microflow regulation can be achieved in response to temperature variations, which can be useful to control the flow rate of a reagent in response to heat production in an on-chip reaction chamber.

\section{REFERENCES}

[1] E.C. Peters, F. Svec, J.M.J. Frechet, Advanced Materials, vol.9, no.8, pp.630-633, 1997.

[2] R.H. Liu, Q. Yu, and D.J. Beebe, JMEMS, vol. 11, no. 1, pp. 45-53, 2002.

[3] B. Stoeber, Z. Yang, D. Liepmann, S. J. Muller, JMEMS, vol. 14, no. 2, pp. 207-213, 2005.

[4] D. D. Joseph, Physics of Fluids, vol. 8, no. 12, pp. 2195-2200, 1965. 